\title{
PTH-independent regulation of blood calcium concentration by the calcium-sensing receptor
}

\author{
Alexandre Loupy, 1,2,3,4 Suresh Krishna Ramakrishnan,1,2,3 Bharath Wootla, 1,2,3,4 \\ Régine Chambrey,1,2,3 Renaud de la Faille,1,3,4,5 Soline Bourgeois, 6 Patrick Bruneval,4,7 \\ Chantal Mandet,4,7 Erik Ilso Christensen, ${ }^{8}$ Hélène Faure, ${ }^{9}$ Lydie Cheval,1,2,3,4 Kamel Laghmani,, $1,2,3,4$ \\ Corinne Collet, ${ }^{10}$ Dominique Eladari,1,3,4,5 Robert H. Dodd,11 Martial Ruat,9 and Pascal Houillier ${ }^{1,3,4,5}$ \\ 1INSERM UMRS 872, Centre de Recherche des Cordeliers, Paris, France. 2Université Pierre et Marie Curie, Paris, France. \\ ${ }^{3}$ CNRS ERL7226, Paris, France. ${ }^{4}$ Université Paris Descartes and Necker Hospital Kidney Transplant Department, Assistance Publique-Hôpitaux de Paris, \\ Paris, France. ${ }^{5}$ Hôpital Européen Georges Pompidou, Département de Physiologie, Assistance Publique-Hôpitaux de Paris, Paris, France. \\ 6University of Zürich, Institute of Physiology, Zurich, Switzerland. 7Hôpital Européen Georges Pompidou, Département de Pathologie, \\ Assistance Publique-Hôpitaux de Paris, Paris, France. ${ }^{8}$ Department of Anatomy, University of Århus, Århus, Denmark. \\ ${ }_{9}^{9}$ Signal Transduction and Developmental Neuropharmacology group, CNRS, UPR-3294, N\&D Laboratory, Institut Albert Fessard, \\ Gif-sur-Yvette, France. ${ }^{10} \mathrm{Hôpital}$ Lariboisière, Biochimie et Biologie Moleculaire, Assistance Publique-Hôpitaux de Paris, Paris, France. \\ ${ }^{11}$ Institut de Chimie des Substances Naturelles, CNRS, Gif-sur-Yvette, France.
}

\begin{abstract}
Tight regulation of calcium levels is required for many critical biological functions. The $\mathrm{Ca}^{2+}$-sensing receptor (CaSR) expressed by parathyroid cells controls blood calcium concentration by regulating parathyroid hormone (PTH) secretion. However, CaSR is also expressed in other organs, such as the kidney, but the importance of extraparathyroid CaSR in calcium metabolism remains unknown. Here, we investigated the role of extraparathyroid CaSR using thyroparathyroidectomized, PTH-supplemented rats. Chronic inhibition of CaSR selectively increased renal tubular calcium absorption and blood calcium concentration independent of PTH secretion change and without altering intestinal calcium absorption. CaSR inhibition increased blood calcium concentration in animals pretreated with a bisphosphonate, indicating that the increase did not result from release of bone calcium. Kidney CaSR was expressed primarily in the thick ascending limb of the loop of Henle (TAL). As measured by in vitro microperfusion of cortical TAL, CaSR inhibitors increased calcium reabsorption and paracellular pathway permeability but did not change $\mathrm{NaCl}$ reabsorption. We conclude that CaSR is a direct determinant of blood calcium concentration, independent of PTH, and modulates renal tubular calcium transport in the TAL via the permeability of the paracellular pathway. These findings suggest that CaSR inhibitors may provide a new specific treatment for disorders related to impaired PTH secretion, such as primary hypoparathyroidism.
\end{abstract}

\section{Introduction}

Calcium is the most abundant mineral in the human body, and its tight regulation is required for many critical biological functions, such as bone mineralization, muscle contraction, nerve conduction, hormone release, and blood coagulation. Extracellular fluid (ECF) Ca concentration is maintained within a narrow range in normal individuals, since any change in Ca entry in the ECF is rapidly matched by an identical change in urinary Ca excretion. Available evidence indicates that adequate secretion of parathyroid hormone (PTH) is absolutely required for the minute-to-minute control of ECF Ca concentration. In accordance with this observation, primary increases or decreases in PTH secretion are responsible for abnormal increases or decreases in blood Ca concentration, respectively (1). Parathyroid cells must appropriately adapt their PTH secretion to the prevailing ECF Ca concentration and, therefore, must be able to sense its level.

A critical step forward in the understanding of Ca homeostasis has been the cloning of the $\mathrm{G}$ protein-coupled extracellular $\mathrm{Ca}^{2+}$-sensing

Authorship note: Alexandre Loupy and Suresh Krishna Ramakrishnan contributed equally to this work.

Conflict of interest: The authors have declared that no conflict of interest exists. Citation for this article: J Clin Invest. 2012;122(9):3355-3367. doi:10.1172/JCI57407. receptor (CaSR) from bovine parathyroid gland (2). The CaSR, which belongs to the family $\mathrm{C}$ (also called family 3 ) of $\mathrm{G}$ protein-coupled receptors (GPCRs), plays a paramount role in the regulation of $\mathrm{Ca}$ homeostasis by modulating PTH secretion of the parathyroid gland. At high levels of extracellular Ca, CaSR inhibits the secretion of PTH through a typical negative short-loop feedback (for review, see ref. 3). The importance of parathyroid CaSR in Ca homeostasis is supported by the chronic hyper- or hypocalcemia observed in patients bearing loss- or gain-of-function mutations, respectively, in the CASR gene (4). Furthermore, drugs targeting CaSR have been shown to ameliorate primary or secondary hyperparathyroidism $(5,6)$.

However, CaSR is expressed in various organs outside the parathyroid glands, particularly in the kidney, where it has been reported to affect tubular $\mathrm{Ca}$, sodium, water, and hydrogen ion transport (3). The importance of extraparathyroid CaSR in the control of $\mathrm{Ca}$ metabolism is difficult to unravel in the intact organism, because of simultaneously occurring changes in PTH release. Double knockout models wherein PTH production was either suppressed or severely reduced were generated to circumvent this complexity $(7,8)$. Data from these models suggest that the extraparathyroid CaSR may limit the variability of blood and urine Ca (8). Moreover, studies in mice have shown that the CaSR in kidney and/or $\mathrm{C}$ cells plays an important homeostatic role in the defense against 

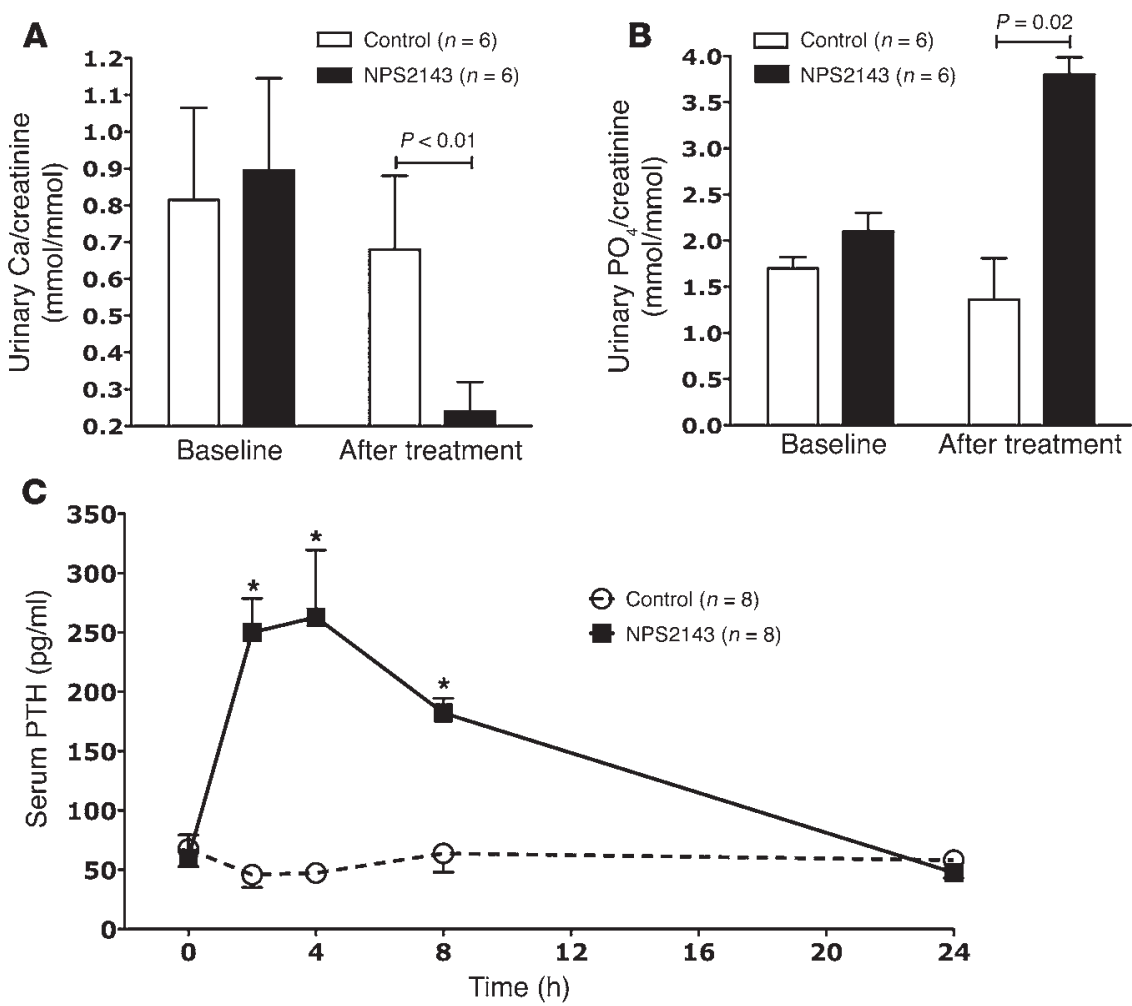

\section{Figure 1}

Effects of a single administration of the CaSR inhibitor NPS2143 in intact rats. (A) Urinary $\mathrm{Ca}$ excretion (expressed as a ratio to urinary creatinine excretion) before (Baseline) and during the 8 hours following a single oral dose of NPS2143 (After treatment) $\left(100 \mu \mathrm{mol} . \mathrm{kg} \mathrm{BW}^{-1}\right)$ or vehicle in intact rats. $n=6$ in each group. (B) Urinary phosphate excretion (expressed as a ratio to urinary creatinine excretion) before and during the 8 hours following a single oral dose of NPS2143 or vehicle in intact rats. $n=6$ in each group. (C) Plasma PTH concentration before (time 0 ) and during the 24 hours following a single oral dose of NPS2143 (black symbols) or vehicle (open symbols) in intact rats. $n=8$ in each group. ${ }^{*} P<0.01$ versus controls. hypercalcemia induced by oral Ca overload or by PTH or calcitriol administration $(9,10)$. However, these experiments did not aim at providing evidence that extraparathyroid CaSR plays a significant role as a direct determinant of blood Ca concentration.

Here, we assessed the effect of acute and chronic inhibition of extraparathyroid CaSR on Ca metabolism. We show that inhibiting renal Ca excretion with a calcilytic increases the serum Ca concentration in parathyroidectomized rats with or without PTH infusion. This study demonstrates that CaSR is a direct determinant of blood Ca concentration independent of PTH. We show that the mechanism involves a modulation of renal tubular Ca transport in the thick ascending limb of the loop of Henle (TAL) via the permeability of the paracellular pathway to $\mathrm{Ca}$. Our data provide evidence that the use of CaSR inhibitors should be considered as a therapeutic option for the care of patients with hypoparathyroid disease.

\section{Results}

CaSR blockade recapitulates the effects of increased PTH secretion. We first tested the in vivo effects in rats of a single oral dose of $100 \mu \mathrm{mol} . \mathrm{kg} \mathrm{BW}^{-1}$ of NPS2143, a CaSR antagonist that was previously characterized (and used it at the same dose as in Gowen et al., ref. 11). NPS2143 induced a significant decrease in the urinary $\mathrm{Ca} /$ creatinine ratio and a concomitant increase in the urinary phosphate/creatinine ratio for 8 hours after drug administration when compared with vehicle treatment (Figure 1, A and B, respectively). The alterations in urinary $\mathrm{Ca}$ and phosphate excretions paralleled a marked increase in serum PTH concentration (Figure 1C), which returned toward baseline values 24 hours following the NPS2143 administration. All other urinary variables (urinary excretion of $\mathrm{Na}$, creatinine, $\mathrm{K}$, bicarbonate, urinary $\mathrm{pH}$, and osmolality) remained unchanged (see Supplemental Table 1; supplemental material available online with this article; doi:10.1172/JCI57407DS1).
Changes in PTH secretion are not required for the effects of CaSR on $\mathrm{Ca}$ metabolism. The effects on urinary $\mathrm{Ca}$ and phosphate excretion observed in the preceding experiments could be explained by changes in PTH concentration. We therefore tested the effects of NPS2143 in rats where PTH concentrations were clamped at normal levels. Rats were thyroparathyroidectomized (TPTX) and supplemented with PTH delivered continuously with osmotic minipumps (Figure 2A). At baseline, the two groups of rats exhibited the same normal blood ionized Ca concentration (1.21 \pm 0.04 vs. $1.23 \pm 0.06 \mathrm{mmol} / \mathrm{l}$ in control and NPS2143-treated groups, respectively, $P=\mathrm{NS}$ ) and urinary Ca excretion (Figure 2, B and C). A dramatic decrease in urinary $\mathrm{Ca}$ excretion occurred in treated animals as compared with controls (Figure 2B) on the first day of NPS2143 administration. Since fecal excretion of $\mathrm{Ca}$ did not change in NPS2143-treated rats (Supplemental Table 2), a positive Ca balance therefore developed on the first day of treatment $(+2.1 \pm 0.8 \mu \mathrm{mol})$. After an initial decrease, urinary $\mathrm{Ca}$ excretion increased back to baseline values and then remained steady (Figure 2B). Similar changes were observed when daily urinary $\mathrm{Ca}$ excretion was considered instead of urinary $\mathrm{Ca} /$ urinary creatinine ratio (Supplemental Table 2). This indicates, together with the sustained increase in blood Ca concentration, that renal Ca handling had changed (fractional excretion of $\mathrm{Ca}$ [FECa] had decreased) and was likely a key participant in sustaining the increase in serum Ca.

We found that dietary intake and fecal Ca excretion in NPS2143treated and control rats did not change throughout the study, indicating that the net intestinal $\mathrm{Ca}$ absorption was not significantly modified by the calcilytic drug and remained similar in the two groups at baseline and throughout the experiment (Supplemental Table 2). In agreement with this finding, plasma 1,25-dihydroxyvitamin D concentrations were similar in control and NPS2143treated rats $(483 \pm 49$ and $348 \pm 47 \mathrm{pmol} / 1$, respectively, $P=\mathrm{NS})$. 

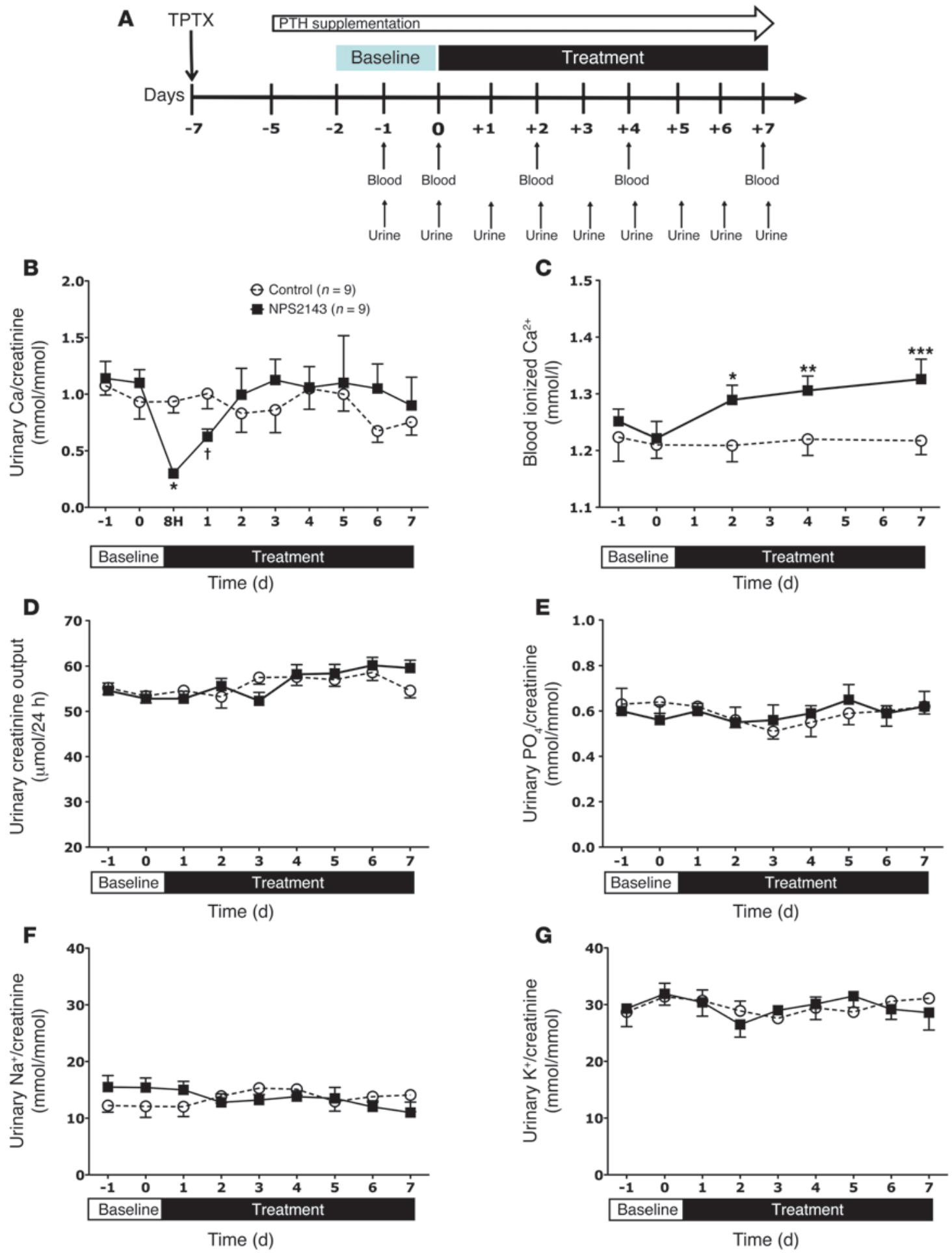

Figure 2

Effects of 1-week daily administration of NPS2143 in TPTX, PTH-supplemented rats. (A) Summary of the protocol. (B) Urinary Ca excretion (expressed as a ratio to urinary creatinine excretion) at baseline and during 1 week of daily administration of NPS2143 (or placebo). In NPS2143treated rats $(1.5 \mathrm{mg} / \mathrm{d}$ intraperitoneally), urinary Ca excretion significantly decreased and was lower than in control rats $(P<0.01)$ on the first day of NPS2143 administration. Subsequently, urinary Ca excretion increased back to baseline values. $n=9$ per group. ${ }^{\star} P<0.01,{ }^{\dagger} P=0.03$ versus control group. (C) Blood ionized Ca concentration at baseline and on days 2, 4, and 7 of daily administration of NPS2143 (or placebo); in treated rats, blood ionized Ca concentration significantly increased by the second day of NPS2143 administration and remained higher than in control rats throughout the period. ${ }^{\star} P=0.05,{ }^{*} P=0.02,{ }^{* \star \star} P=0.01$. (D-G) Twenty-four-hour urinary creatinine excretion and urinary phosphate, Na, and $\mathrm{K}$ excretion (expressed as a ratio to urinary creatinine excretion) at baseline and during 1 week of daily administration of NPS2143 (or placebo). No statistical difference between treated and control rats was seen for any variable. $n=9$ per group. 


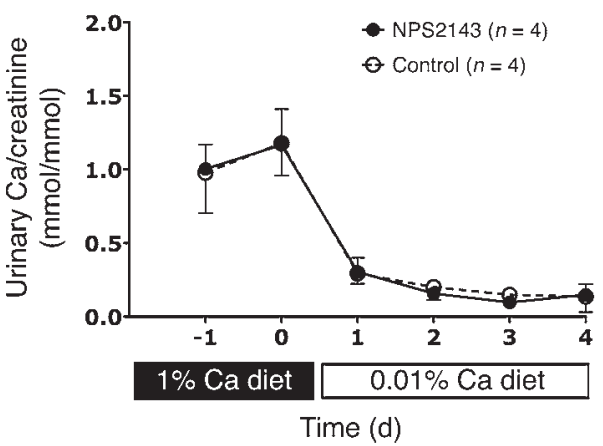

Urinary output, creatinine, phosphate, $\mathrm{Na}$, and $\mathrm{K}$ urinary excretion and osmolality were notably unaffected by CaSR inhibition (Figure 2, D-G, and Supplemental Table 2). Similarly, plasma creatinine concentrations were the same in control and NPS2143treated rats (Supplemental Table 2).

We then measured urinary Ca excretion in control and NPS2143treated rats on a low-Ca diet. Under this condition, where urinary $\mathrm{Ca}$ excretion reflects net bone Ca release, no difference was observed between control and NPS-treated rats (Figure 3). In this experiment, there was a clear trend toward a higher serum ionized Ca concentration in NPS2143-treated rats $(1.17 \pm 0.01$ as compared with $1.12 \pm 0.02 \mathrm{mM}$ in control rats at day $4[P=0.058])$, indicating that "normal" intestinal absorption of Ca may not be required for the effect of the CaSR inhibitor on blood Ca concentration.

Furthermore, two bone remodeling markers, blood osteocalcin concentration $(57 \pm 8$ and $54 \pm 3 \mathrm{ng} / \mathrm{ml}$ in control and treated rats, respectively) and urinary deoxypyridinoline excretion (293 \pm 46 and $276 \pm 26 \mathrm{nmol} / \mathrm{mmol}$ in control and NPS2143-treated rats, respectively) did not differ between the two groups (Supplemental Table $2)$. We then treated TPTX, PTH-supplemented rats with an antibone-resorptive agent $\left(2.5 \mu \mathrm{g}\right.$ pamidronate. $\mathrm{BW}^{-1}$, daily for 4 days prior to the experiment); this treatment significantly inhibits bone resorption in rats and mice as validated in previous studies $(9,12$, 13). As shown in Figure 4, at baseline, both groups of rats exhibited the same blood ionized Ca concentration and urinary Ca excretion. By the first day of treatment with NPS2143, and throughout the remainder of the experiment, the urinary excretion of Ca was lower than at baseline, whereas it did not change in control rats. Conse-

\section{Figure 4}

Effect of NPS2143 in TPTX, PTH-supplemented rats treated with the anti-bone-resorptive agent sodium pamidronate. All rats were treated with subcutaneous injections of pamidronate $\left(2.5 \mu \mathrm{g} . \mathrm{g} \mathrm{BW}^{-1}\right.$, daily for 4 days prior to the experiment and throughout the remainder of the experiment). At baseline, the two groups of rats exhibited similar blood ionized $\mathrm{Ca}$ concentration and urinary $\mathrm{Ca}$ excretion. (A) During treatment with NPS2143, urinary Ca excretion decreased by the first day of treatment; throughout the remainder of the experiment, the NPS-treated rats exhibited significantly lower urine $\mathrm{Ca}$ excretion as compared with control rats. ${ }^{*} P=0.01$ versus controls; ${ }^{\dagger} P=0.02$ versus baseline. (B) In contrast to control rats in which blood Ca concentration did not change during the experiment, blood ionized Ca significantly increased in NPS2143-treated rats by day $7\left({ }^{\star} P=0.023\right.$ and ${ }^{* \star} P=0.012$ at day 7 and day 9 , respectively, as compared with day 0 ). This result indicates that even when bone resorption is inhibited, NPS2143 induces an increase in renal tubular $\mathrm{Ca}$ absorption and a positive $\mathrm{Ca}$ balance, resulting in a secondary increase in blood Ca concentration. $n=5$ in the NPS2143-treated group and $n=4$ in the control group.

\section{Figure 3}

Effects of a low-Ca diet in NPS2143-treated or control TPTX, PTHsupplemented rats. Shown is the effect of a low-Ca $(0.01 \%, w / w)$ diet as compared with a normal $\mathrm{Ca}$ diet on urinary $\mathrm{Ca}$ excretion in TPTX, PTH-supplemented rats treated with a CaSR inhibitor or vehicle alone. The rats received either NPS2143 (1.5 mg daily) or vehicle for 4 days before the switch while on the regular Ca diet. The switch to the lowCa diet induced a similar decrease in urinary $\mathrm{Ca} /$ creatinine ratio in NPS2143-treated and control rats $(P=\mathrm{NS})$. This result indicates that net bone $\mathrm{Ca}$ release was not affected by treatment with the CaSR inhibitor. $n=4$ in each group.

quently, the NPS2143-treated rats exhibited significantly lower urinary Ca excretion than control rats $(P<0.01)$. In contrast to control rats, in which blood Ca concentration did not change during the experiment, blood ionized Ca significantly increased in NPS2143treated rats $(P=0.016)$. This indicates that even when bone resorption is inhibited, NPS2143 induced an increase in renal tubular $\mathrm{Ca}$ absorption and a positive extracellular Ca balance, resulting in a secondary increase in blood Ca concentration. That urinary $\mathrm{Ca}$ excretion remained lower in NPS2143-treated than in control rats throughout the period suggests that inhibiting CaSR induced an inward Ca flux to bone when bone resorption was already inhibited by the bisphosphonate.

Taken together, these results indicate that CaSR blockade affects renal tubular $\mathrm{Ca}$ handling. CaSR inhibition in rats with a normal but clamped PTH supply had no effect on intestinal Ca absorption or glomerular filtration rate, did not increase bone Ca release but increased renal tubular Ca reabsorption sufficiently to create a sustained increase in blood Ca concentration.
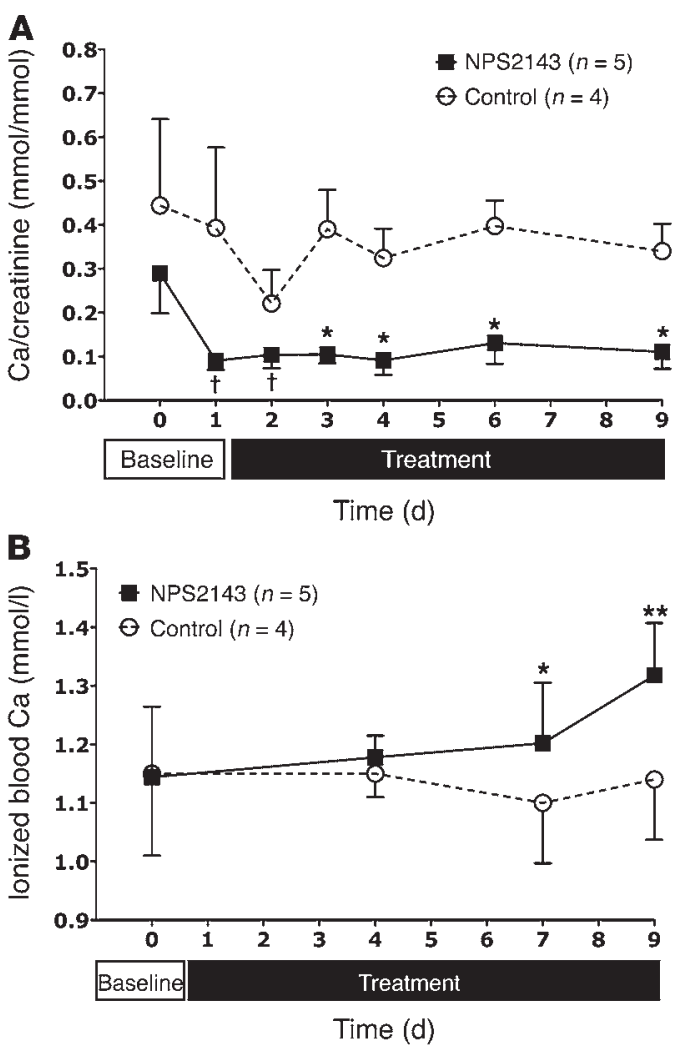


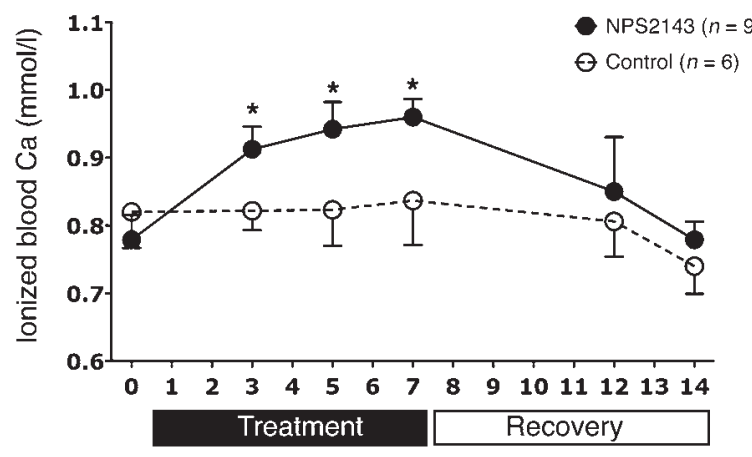

NPS2143 increases blood Ca concentration in the nominal absence of PTH. It is conceivable that an alteration of the sensitivity of the PTH receptor or of its downstream signaling pathway(s) accounts for the effects of CaSR blockade observed in the PTHsupplemented rats. To test this possibility, we assessed the effects of NPS2143 on Ca homeostasis in the nominal absence of PTH secretion. Rats were TPTX but not supplemented with PTH. As expected, all animals became and remained hypocalcemic after the TPTX procedure (Figure 5). However, when the rats were administered NPS2143, serum ionized Ca concentration increased and stabilized at a significantly higher value than in control rats (Figure 5). At steady state, urinary Ca excretion was similar in the NPS2143-treated and the control groups

\section{Figure 5}

Effects of 1-week daily administration of NPS2143 in TPTX rats without PTH supplementation. The time course of blood Ca concentration during 1-week treatment of TPTX rats with either vehicle or NPS2143 is summarized. At baseline, both groups had low blood Ca concentration. In the control group, serum Ca concentration remained unchanged throughout the experiment, while a significant and sustained increase in blood Ca concentration was observed in the NPS2143-treated group ( ${ }^{\star} P<0.02$ as compared with day 0 ). After a 7 -day recovery period, serum Ca concentration returned to baseline values. $n=9$ and $n=6$ in the NPS2143-treated and control groups, respectively.

$(0.046 \pm 0.01$ vs $0.044 \pm 0.001 \mathrm{mmol} / \mathrm{mmol}$ creatinine, respectively, $P=\mathrm{NS}$ ); because the serum Ca concentration was higher in the NPS2143-treated rats, these results indicate that FECa had decreased. These results demonstrate that even in the nominal absence of PTH, NPS2143 can stimulate renal tubular Ca absorption, which sustains the drug-induced increase in blood Ca concentration. After 7 days of treatment withdrawal (day 7 to day 14), blood ionized Ca concentration returned toward similarly low values in both groups (Figure 5).

CaSR expression within the kidney is restricted to the TAL. The results obtained in vivo indicate that a CaSR antagonist alters blood $\mathrm{Ca}$ mainly through changes in the renal handling of $\mathrm{Ca}$. Previously documented conflicting results reported with regards to CaSR
A

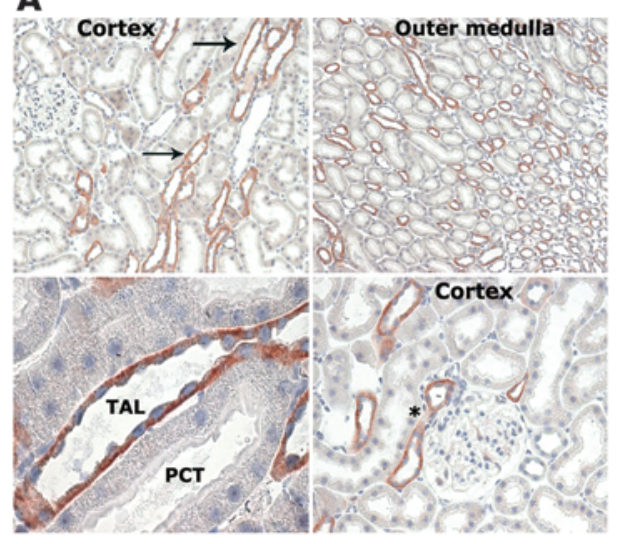

B

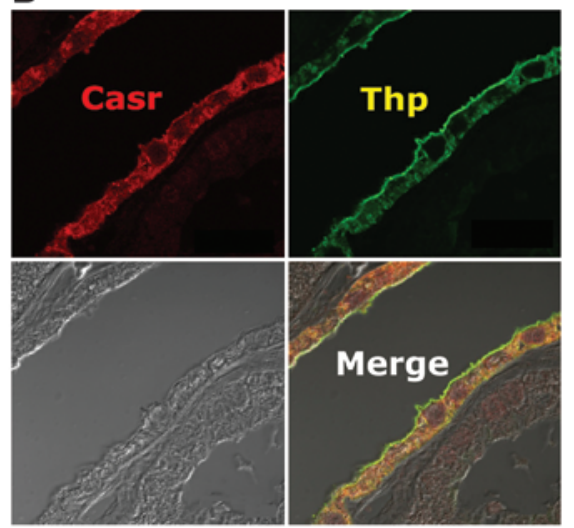

C

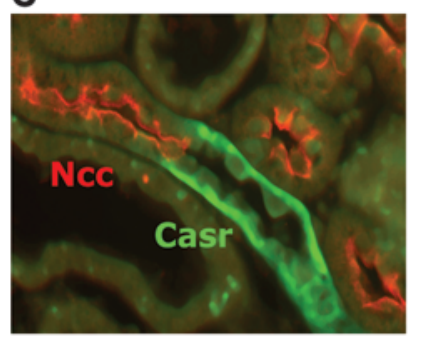

D

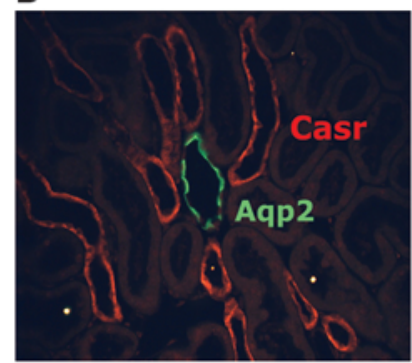

\section{Figure 6}

Immunolocalization of CaSR in the rat kidney. (A) Immunolocalization of the CaSR protein in the renal cortex and outer medulla. Immunoperoxidase staining in rat kidney sections showed a cytoplasmic and basolateral labeling of tubular cells in the cortex (original magnification, $\times 200$ and $\times 400)$ and the inner and outer stripes of the outer medulla $(\times 100)$. The stained tubules were present in the medullary rays (arrows) and in the juxtaglomerular apparatus (asterisk). Proximal tubules and glomeruli showed no detectable CaSR staining $(\times 1,000)$. (B) CaSR is expressed in the rat TAL. Shown is double staining with Abs directed against CaSR (red) and Tamm-Horsfall protein (Thp), specifically expressed in the TAL (green). When comparing with tubular segments that stained for Tamm-Horsfall protein, CaSR staining was basolateral (original magnification, $\times 2,000$ ). (C) CaSR is not detectable in the rat distal convoluted tubule (DCT). Sections of rat kidney double stained for CaSR and the thiazidesensitive $\mathrm{NaCl}$ cotransporter (Ncc) localized in the DCT. Basolateral CaSR staining was interrupted when the tubule showed apical staining for $\mathrm{Ncc}$, thus representing the distal limit for CaSR staining (original magnification, $\times 1,500)$. (D) CaSR is not detectable in the rat CCD. Sections of rat kidney double stained for CaSR and aquaporin-2 (Aqp2). In the merged image, tubular segments that stained for aquaporin-2 did not show CaSR staining and vice versa (original magnification, $\times 1,000$ ). 


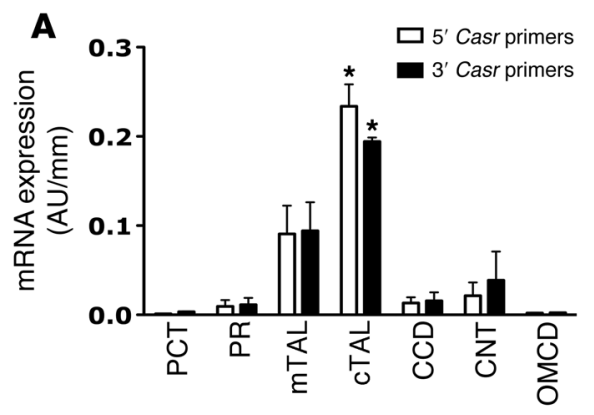

B

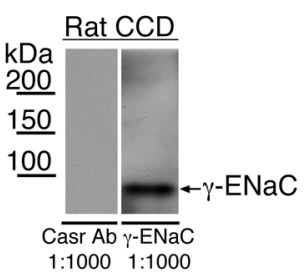

c

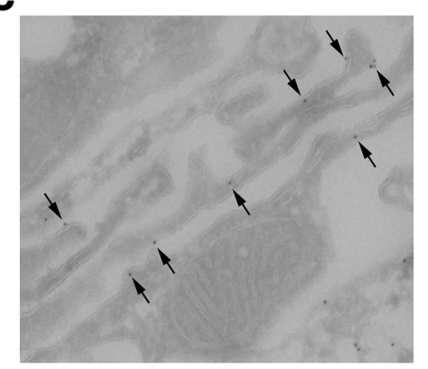

Figure 7

Localization of CaSR in the rat kidney. (A) Quantification of Casr transcripts along the rat nephron. RT-PCR analysis of mRNAs encoding CaSR was performed on microdissected renal tubules. Casr transcripts were detected at a significant level in the medullary TAL (mTAL) and CTAL. No significant transcript expression was detected in the PCT, PR, CCD, or OMCD. A non-significant level of Casr mRNAs was found in the CNT. ${ }^{*} P<0.001$ between cTAL and mTAL Casr mRNA expression. (B) Western blotting for CaSR in rat microdissected CCD, using 1:1,000 anti-CaSR Ab (MA1-934). No signal was observed using anti-CaSR Ab. $\gamma$-ENaC (specific for CCD) was used as a positive control. (C) CaSR localization in the kidney by EM. CaSR immunocytochemistry of ultrathin cryosections from rat kidney with gold labeling for CaSR in the TAL showing basolateral gold labeling (arrow). No apical TAL labeling and no CCD labeling were noted (original magnification, $\times 100,000$ ).

expression along the nephron (14-16) have been confusing. This led us to determine CaSR expression in the kidney of 3 species (rats, mice, and humans), using four complementary approaches.

We first performed CaSR immunolocalization in the rat kidney using two different anti-CaSR Abs, one recognizing an epitope located in the $\mathrm{N}$-terminal end of the protein and the other directed against a distinct epitope located in the $\mathrm{C}$-terminal tail of the protein. Both Abs were checked in detail for specificity in preliminary experiments (Supplemental Figures 1 and 2). Proximal tubules were identified by the presence of a brush border, and co-staining with specific markers was performed to unambiguously identify the various segments from the distal nephron. Figure 6A shows that CaSR immunostaining was restricted to renal tubules extending from the cortex to the inner stripe of the outer medulla, which were identified as TAL by co-staining with an Ab directed against Tamm-Horsfall protein, a TAL-specific protein (Figure 6B). CaSR staining was abruptly interrupted at the beginning of the distal convoluted tubule as identified by an $\mathrm{Ab}$ directed against the $\mathrm{NaCl}$ cotransporter $\mathrm{Ncc}$ (Figure 6C). No CaSR staining was found in the collecting duct as indicated by aquaporin-2 labeling (Figure 6D). Glomeruli, interstitium, and arteries stained negative. Importantly, the same localization was observed in mouse and human kidney sections using Ab10-12 and MA1-934 Abs
(Supplemental Figures 3 and 4). As a second approach, we studied the expression level of Casr transcripts in microdissected rat tubules. Casr transcripts were detected at a significant level exclusively in medullary and cortical TAL (cTAL) (Figure 7A), whereas Casr transcript was not detected at a significant level in the proximal convoluted tubule (PCT), in the pars recta (PR), in the connecting duct $(\mathrm{CNT})$, or in the cortical or outer medullary collecting duct (CCD and OMCD).

In a third approach, we tested CaSR expression by immunoblot technique on proteins extracted from isolated rat CCDs. Figure 7B shows Western blotting for CaSR in rat microdissected CCD, using 1:1,000 anti-CaSR Ab (MA1-934). No signal was observed using anti-CaSR Ab. $\gamma$-ENaC (specific for CCD) was used as a positive control. Anti-CaSR Abs (Ab10-12) in mice reacted with a 150-kDa polypeptide in TAL, whereas no signal was detected in protein extracted from isolated CCDs (Supplemental Figure 5). EM immunocytochemistry revealed labeling of the TAL basolateral membrane (Figure 7C).

In a fourth and final approach, to confirm that CaSR expression is restricted to TAL, we assessed the CaSR functional expression by studying the Ca signaling response to the polyvalent cation neomycin and to NPS-R568, two CaSR agonists, in either CTAL or CCD, isolated and microperfused in vitro. Peritubular addition of $1 \mu \mathrm{M}$ NPS-R568 or $300 \mu \mathrm{M}$ neomycin elicited a significant increase in cytosolic free $\mathrm{Ca}^{2+}$ concentration in microperfused TAL (Supplemental Figure 6A). In contrast, neither peritubular nor luminal addition of the same agonists induced any significant response in microperfused CCDs (Supplemental Figure 6, B and C). Taken together, our results demonstrate that the localization and functional expression of CaSR in the kidney are restricted to the TAL.

CaSR controls paracellular pathway permeability to Ca in the TAL. To determine the cellular mechanism of action of CaSR on Ca transport, cTALs were isolated from rats under normal conditions and perfused in vitro for analysis of the effect of $1 \mu \mathrm{M}$ NPS2143 on $\mathrm{Ca}^{2+}, \mathrm{Na}^{+}$, and $\mathrm{Cl}^{-}$transepithelial flux $\left(\mathrm{J}_{\mathrm{Ca}}, \mathrm{J}_{\mathrm{Na}}\right.$, and $\mathrm{J}_{\mathrm{Cl}}$, respectively) and on transepithelial voltage (Vte). The peritubular addition of $1 \mu \mathrm{M}$ NPS2 143 increased $\mathrm{J}_{\mathrm{Ca}}$ (Figure 8), this effect being reversed when NPS2143 was removed from the bath. By contrast, NPS2143 did not alter net transepithelial $\mathrm{Na}^{+}$flux, $\mathrm{Cl}^{-}$flux, ot Vte.

These results confirm that CaSR blockade increases Ca absorption by cTAL, demonstrating that CaSR exerts negative control on Ca transport and that blood Ca concentration directly regulates renal Ca transport. In the TAL segment, Ca transport is paracellular and is driven by the lumen-positive Vte generated by the transcellular absorption of $\mathrm{NaCl}$. Since neither $\mathrm{NaCl}$ absorption nor Vte were affected by NPS2143, we hypothesized that CaSR exerts its effects on Ca transport by altering the permeability of the paracellular pathway to Ca. To further confirm this hypothesis, we directly measured the paracellular pathway permeability to $\mathrm{Ca}$ in microdissected cTALs that were treated by peritubular addition of NPS2143. Interestingly, the CaSR inhibitor increased the paracellular pathway permeability to Ca by $41 \%$ (from $6.9 \times 10^{-5} \pm 0.6 \times 10^{-5}$ to $9.7 \times 10^{-5} \pm 0.9 \times 10^{-5} \mathrm{~cm} . \mathrm{s}^{-1}$ in the absence and presence of peritubular NPS2143, respectively (Figure 8D, $P=0.0027$ ).

In order to determine whether the effect of CaSR inhibition would be similar in the presence of PTH, we tested the effect of the calcilytic NPS2143 on the Ca reabsorption in cTAL bathed with a solution containing 300 pM PTH (Figure 8E). As expected, the Ca reabsorption was higher in the presence of peritubular PTH than 


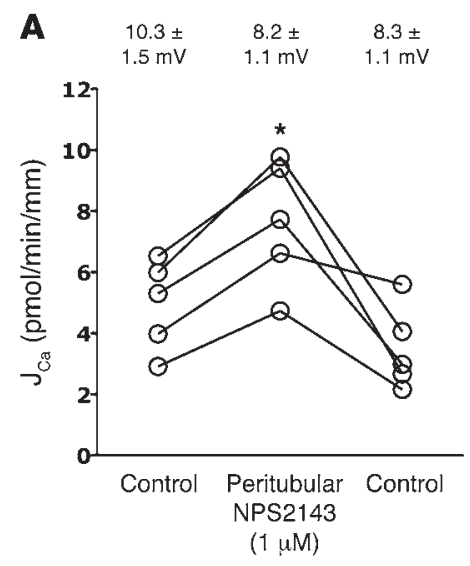

D

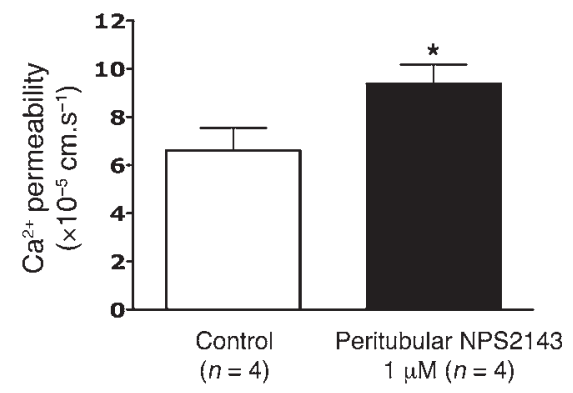

B

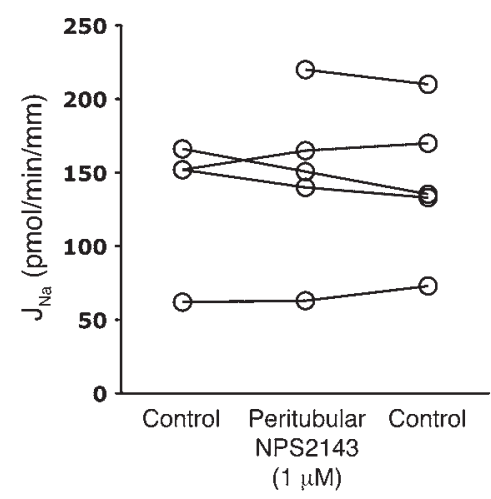

C

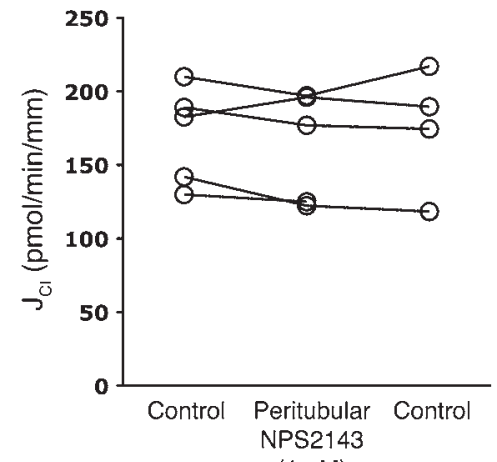

$(1 \mu \mathrm{M})$

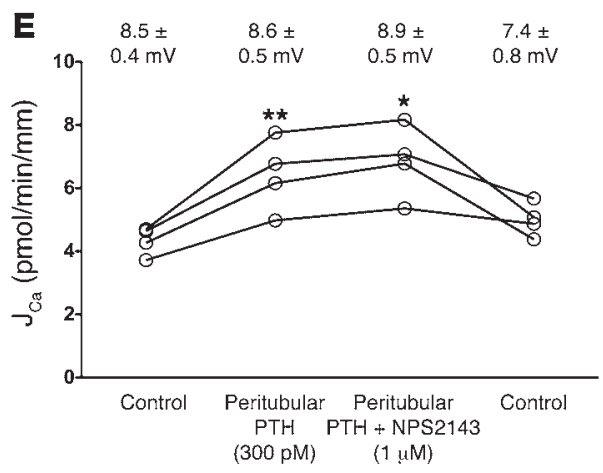

\section{Figure 8}

Effect of NPS2143 on transepithelial ion transport in the rat cTAL. cTALs were dissected from rat kidney and perfused in vitro as explained in Methods. Peritubular addition of $1 \mu \mathrm{M}$ NPS2143 elicited a significant and reversible increase in transepithelial Ca absorption ( $\left.{ }^{\star} P=0.03\right)$, but did not change Vte $(\mathbf{A})$ or transepithelial sodium or chloride absorption (B and $\mathbf{C}$, respectively) $(P=\mathrm{NS}) . n=5$. (D) $1 \mu \mathrm{M}$ NPS2143 elicited a significant increase in the paracellular pathway permeability to Ca in rat cTAL microperfused in vitro ( $\left.{ }^{\star} P=0.0027\right) . n=4$. (E) As compared with the control period, PTH (300 pM in the peritubular fluid) elicited a significant increase in transepithelial Ca absorption (** $P=0.024)$; peritubular addition of $1 \mu \mathrm{M}$ NPS2143, in the presence of PTH elicited a further increase in transepithelial Ca absorption $\left({ }^{*} P=0.045\right.$ as compared with the PTH period) that was significantly lower than in the absence of peritubular PTH $(n=4)$. Vte was not changed throughout the experiment.

in its absence. The calcilytic-induced increase in Ca absorption was significantly lower when NPS2143 was added in the presence of PTH than in its absence, suggesting that the PTH receptor and CaSR act on the same pathway.

Long-term PTH-independent CaSR blockade decreases the renal expression of CaSR without modifying $\mathrm{Nkcc} 2$ and $\mathrm{Na}^{+}, \mathrm{K}^{+}$-ATPase expression. The above experiments studied the short-term effect of CaSR inhibition in the CTAL. To assess whether long-term CaSR inhibition might affect the expression of transport proteins in the TAL, we studied the expression of the apical $\mathrm{NaCl}$ cotransporter $\mathrm{Nkcc} 2$, the basolateral $\mathrm{Na}^{+}, \mathrm{K}^{+}$-ATPase $\alpha_{1}$ subunit, as well as of CaSR proteins in membrane preparations of the renal cortex of TPTX, PTH-supplemented rats treated with NPS2143 or vehicle. Following a 7-day treatment, we found that the expression of CaSR was dramatically reduced, by $64 \%$, in treated as compared with control rats $(P<0.01$, Figure 9). In contrast, the expression of $\mathrm{Nkcc} 2$ and $\mathrm{Na}^{+}, \mathrm{K}^{+}$-ATPase $\alpha_{1}$ subunit was not modified by treatment with the CaSR inhibitor (Figure 9). Finally, we measured the expression of CaSR in TPTX rats without PTH supplementation. The expression of CaSR was not altered in hypocalcemic, TPTX rats as compared with normocalcemic, TPTX, PTH-supplemented rats (Figure 10). This suggests that hypocalcemia alone is not able to induce a measurable change in CaSR expression in the TAL.

\section{Discussion}

The role of CaSR expressed outside the parathyroid glands on mineral metabolism remains unclear. The generalized disruption of Casr in the mouse has failed to provide much information, owing to the lethality of the model and its association with hyperparathyroidism (17). To circumvent these drawbacks, researchers have bred $\mathrm{Casr}^{-1}$ - mice with mice lacking parathyroid glands (7) or unable to synthesize PTH (8). In studies in the latter model, it was proposed that extraparathyroid CaSR was useful for narrowing the variability of blood and urine $\mathrm{Ca}(8)$ and dampening the calcemic response to exogenous PTH administration, high-Ca diet, or calcitriol injections $(9,10)$. However, these studies did not aim at providing direct evidence that extraparathyroid $\mathrm{Ca}$ is important in determining the value of steady-state blood Ca concentration when PTH is either absent or invariant.

In this study, we have eliminated endogenous PTH (and calcitonin) secretion by surgically removing the parathyroid and thyroid glands, thereby ensuring that the effects of CaSR inhibitors are not mediated by changes in PTH and calcitonin secretion. Additionally, this approach allowed us to describe both the short- and long-term effects of CaSR inhibitors on Ca metabolism. One of the major findings of this study is that CaSR antagonists can elicit a sustained increase in plasma Ca concentration in rats with 
A

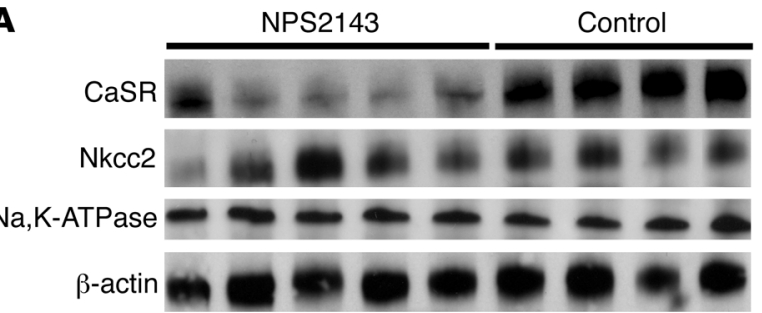

B
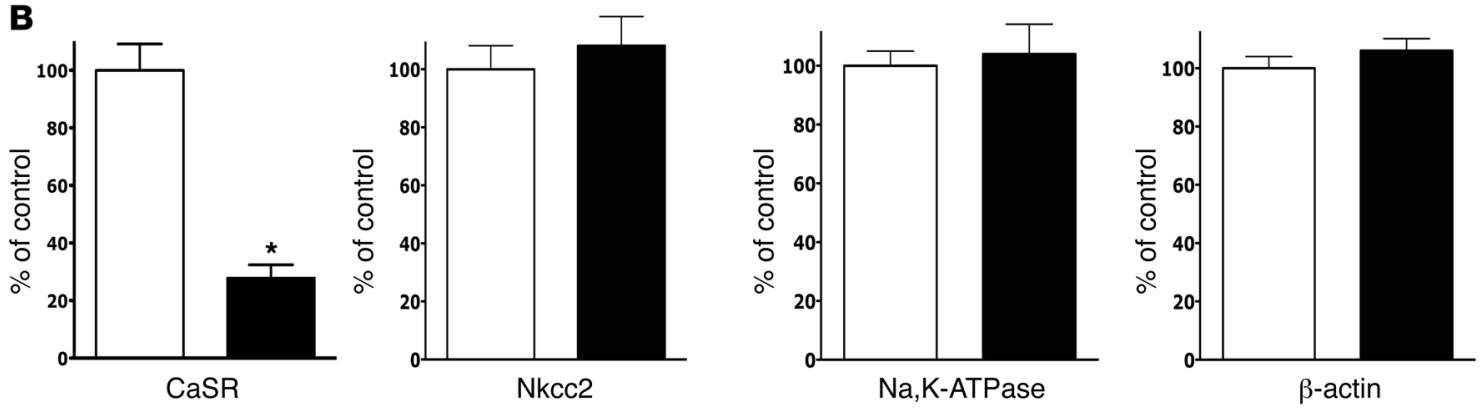

\section{Figure 9}

Effect of a 7-day treatment with NPS2143 on CaSR, Nkcc2, and $\mathrm{Na}^{+}, \mathrm{K}^{+}$-ATPase $\alpha_{1}$ subunit protein abundance in the rat kidney cortex. (A) Immunoblots of membrane fractions from the rat kidney cortex obtained from 5 NPS2143-treated and 4 control rats. Immunoblots were reacted with Abs directed against CaSR, Nkcc2, the $\alpha_{1}$ subunit of the $\mathrm{Na}^{+}, \mathrm{K}^{+-}$-ATPase, and $\beta$-actin. (B) Western blot analyses showing the abundance of CaSR, Nkcc2, and the $\alpha_{1}$ subunit of the $\mathrm{Na}^{+}, \mathrm{K}^{+}-$ATPase in NPS2143-treated rats (black bars) and in control rats (white bars). CaSR expression was quantified by densitometric analysis and expressed as percentage of control values. ${ }^{*} P<0.001$ versus controls. Nkcc2, Na+, $\mathrm{K}^{+}-\mathrm{ATPase} \alpha_{1}$ subunit, and $\beta$-actin expression was not modified by the treatment.

invariant or no PTH. Plasma Ca concentration, once raised, does not change as long as the CaSR antagonist is administered. This corresponds to one type of equilibrium increase in plasma $\mathrm{Ca}$ concentration, as defined by Parfitt (18). The prototype of equilibrium hypercalcemia is primary hyperparathyroidism, in which hypercalcemia is due to a PTH-elicited increase in renal tubular Ca reabsorption and, to a lesser extent, in net bone Ca resorption (19). In the present study, the CaSR antagonist administered to TPTX, PTH-supplemented rats elicited a transient initial decrease in urinary $\mathrm{Ca}$ excretion. Given that the glomerular filtration rate was not altered by the CaSR antagonist, this change in urinary $\mathrm{Ca}$ excretion corresponds to an increase in renal tubular Ca reabsorption, which creates a positive Ca balance of sufficient magnitude in order to explain the increase in plasma Ca concentration. The increase in plasma Ca concentration is thus not due to an increase in bone resorption. First, the markers of bone remodeling were not affected by treatment with the CaSR antagonist. Second, the experiment using the bone anti-resorptive agent pamidronate indicated that even when bone resorption is inhibited, NPS2143 still induces an increase in renal tubular Ca absorption and a positive Ca balance, resulting in a secondary increase in blood Ca concentration. Third, the steady-state urinary Ca excretion in rats fed

\section{Figure 10}

Effect of a 7-day hypocalcemia on CaSR protein abundance in the rat kidney cortex. (A) Immunoblots of membrane fractions from the rat kidney cortex obtained from 4 TPTX, PTH-supplemented and 5 TPTX rats. Immunoblots were reacted with Abs directed against CaSR. (B) Western blot analyses showing the abundance of CaSR in TPTX, PTH-supplemented and TPTX, non-PTH-supplemented rats. CaSR expression was quantified by densitometric analysis and expressed as percentage of control values. a very low-Ca diet was the same whether or not they were treated with NPS2143, indicating that NPS2143 does not affect net bone Ca release. Similarly, the increase in plasma Ca concentration was not due to enhanced intestinal Ca absorption, as demonstrated by the steadiness of fecal Ca loss.

Taken together, these results indicate that the CaSR antagonists alter the renal tubular handling of $\mathrm{Ca}$, but not intestinal Ca absorption. They also do not affect bone remodeling and net bone Ca release; however, when bone resorption is inhibited by a bisphosphonate, they elicit an inward flux of Ca to bone.

These data are in agreement with Parfitt's statement that a raised plasma Ca concentration can be maintained indefinitely without losses or gains of Ca from bone (18). This also indicates that a

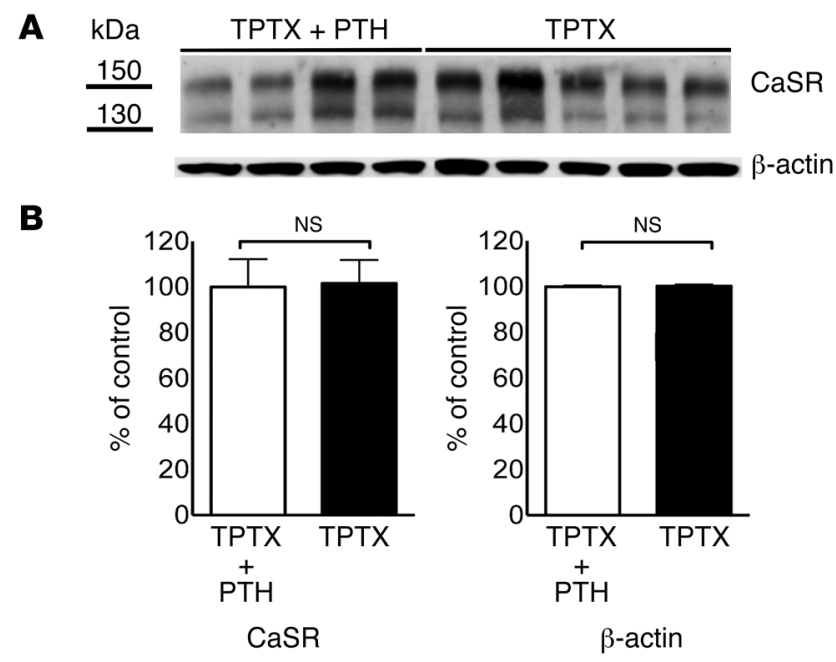


new equilibrium has to be reached between plasma Ca and bone in animals treated with CaSR antagonists, a phenomenon that, according to Parfitt, involves bone-lining cells and osteocytes but not osteoblasts or osteoclasts (18). Finally, our results may explain why patients with loss-of-function mutations in CASR, who display both equilibrium hypercalcemia and normal PTH concentration, have a normal bone mineral mass $(20,21)$.

The last part of the study was directed at dissecting the mechanism by which CaSR acts on the renal tubule. We show here that CaSR in the kidney is exclusively restricted to the TAL. (Previous reports have shown evidence that extracellular, luminal $\mathrm{Ca}$, or polycations are able to decrease the permeability of the collecting duct epithelium to water; ref. 22.) Consistent with this finding, that high extracellular Ca concentration is able to induce polyuria has repeatedly been demonstrated $(23,24)$. Similarly, high extracellular $\mathrm{Ca}$ concentration increases acid secretion in the collecting duct (25). These important data support the concept that a Ca-sensing mechanism does exist in the CCD. Preliminary results from our group suggest that another GPCR, Gprc6a, which belongs to the same subfamily as CaSR, may be such a Ca-sensing protein in the CCD, but additional experiments are needed to confirm these data.

It has been previously proposed that CaSR present along the TAL is not primarily a modulating factor of Ca transport, but rather alters $\mathrm{NaCl}$ absorption and Vte and, hence, indirectly affects $\mathrm{Ca}$ absorption by changing the electromotive force that drives $\mathrm{Ca}$ through the paracellular pathway. According to this model, an increase in extracellular Ca concentration would decrease both $\mathrm{NaCl}$ absorption by the TAL and Vte, and thereby increase urinary $\mathrm{NaCl}$ and, subsequently, Ca excretion. In this respect, increased peritubular Ca concentration and neomycin have been reported to elicit a decrease in $\mathrm{NaCl}$ reabsorption through the in vitro perfused cTAL (26). However, this effect has not been reproduced with more specific CaSR activators.

The results reported here show that antagonizing CaSR does not lead to the opposite effects, i.e., to increased tubular $\mathrm{NaCl}$ absorption and decreased $\mathrm{NaCl}$ urine output. This is in accordance with the results of Desfleurs et al., showing that, in in vitro perfused CTAL, increases in ECF Ca concentration altered neither the Vte nor transepithelial $\mathrm{NaCl}$ reabsorption (27).

One important aspect of the present study is that it helps to elucidate the mechanism by which CaSR directly acts on the renal tubule. We found that CaSR antagonists acutely and directly increase the paracellular pathway permeability of the TAL to Ca, as shown in in vitro microperfused TAL experiments. We therefore propose that CaSR directly inhibits paracellular pathway permeability to Ca. Notably, at the dose used in this study, PTH also increases $\mathrm{Ca}$ absorption without altering the Vte; this confirms that PTH also increases the paracellular pathway permeability to $\mathrm{Ca}$, as already demonstrated by others (28). The effect of the CaSR antagonist on $\mathrm{Ca}$ absorption is lower, however, in the presence than in the absence of PTH, suggesting that PTH and CaSR act on the same target(s).

We also show that chronic treatment with CaSR antagonists decreases the expression of CaSR in the TAL without affecting the expression of proteins involved in $\mathrm{NaCl}$ transport ( $\mathrm{Nkcc} 2$ and $\mathrm{Na}^{+}, \mathrm{K}^{+}$-ATPase). In accordance with this finding, another study suggests that the permeability to cations of the paracellular pathway can be regulated selectively (29). These observations support the view that not only the transcellular but also the paracellular pathway can be specifically gated and dynamically regulated. Of note, we find that chronic hypocalcemia by itself is unable to decrease CaSR abundance in the TAL. This probably explains why profound hypocalcemia can develop in the absence of PTH. This might also explain why the CaSR antagonist NPS2143, which is able to decrease CaSR expression, is able to partially correct hypocalcemia under this condition.

In summary, our results demonstrate for the first time to our knowledge that extraparathyroid CaSR is a factor participating in the homeostasis of ECF Ca independent of PTH. The underlying mechanism mainly involves the TAL, in which an increase in $\mathrm{Ca}$ reabsorption occurs through a specific increase in the paracellular pathway permeability to Ca.

Importantly, this study provides evidence that modulating CaSR activity may help to improve the control of ECF Ca concentration even when PTH secretion is either absent or invariant. In support of this, we demonstrate that the severe hypocalcemic phenotype of TPTX animals can be at least partially reversed by the CaSR antagonist NPS2143.

Since current therapy for hypoparathyroidism, which is mostly based upon administration of high doses of active vitamin D metabolite and Ca salts, often leads to complications such as nephrocalcinosis, kidney stones, and ultimately chronic renal failure, our findings may pave the way for significant therapeutic improvement.

\section{Methods}

\section{Animals}

Pathogen-free male Sprague-Dawley rats (Charles River Laboratories), weighing 250-300 g were used for this study, except in the in vitro microperfusion experiments, for which rats weighing $70-80 \mathrm{~g}$ were used. All animals had free access to deionized water and were fed, ad libitum, standard laboratory chow (M20, Dietex) containing 1.085\% (w/w) Ca, except in the studies in which animals were switched to a low-Ca diet (0.01\% [w/w], TD.95027, Harlan).

\section{Compounds}

$N$-[(R)-2-hydroxy-3-(2-cyano-3-chlorophenoxy)propyl]-1,1-dimethyl-2-(2naphthyl) ethylamine (NPS2143) was from R.H. Dodd (Institut de chimie des substances naturelles, CNRS, Gif-sur-Yvette, France) and previously characterized (11). NPS-R568 was provided by Amgen. All other chemicals were from Sigma-Aldrich.

\section{Animal studies}

In vivo studies were performed in animals housed in metabolic cages (Techniplast). Animals were allowed to adapt to the cages for 3-5 days before starting the study.

Effect of a single administration of NPS2143 in intact rats. During the acute studies, rats had no access to food between 10:00 a.m. and 6:00 p.m. After rats had adapted to the cage, a 2-day experiment was initiated during which urine was collected at 10:00 a.m. and 6:00 p.m. On the second day, just after the morning urine collection, rats received either $100 \mu \mathrm{mol} / \mathrm{kg}$ BW of NPS2 143 in a $20 \%(\mathrm{w} / \mathrm{v}) 2$-hydroxypropyl- $\beta$-cyclodextrin vehicle solution (study group) or vehicle alone (control group) by gastric gavage.

Effect of chronic administration of NPS2143 in TPTX rats. TPTX was carried out in anesthetized rats by peritoneal injection of a mixture $(0.1 \mathrm{ml} / 100 \mathrm{~g} \mathrm{BW})$ of ketamine (Imalgene; 10\% [v/v]) and xylazine (Rompun, Bayer; 5\% $[\mathrm{v} / \mathrm{v}])$. Both thyroid lobes and parathyroid glands were surgically removed (30). The efficiency of the parathyroidectomy was subsequently confirmed by plasma PTH concentration measurement. Unless otherwise specified, all rats were subsequently supplemented with PTH (rat 
1-34 PTH, Bachem) dissolved in sterile saline containing $2 \%(\mathrm{w} / \mathrm{v})$ cysteine chloride monohydrate (Fluka). The PTH-Cys solution was placed in osmotic minipumps (Alzet, no. 2002) delivering 1-34 PTH at a rate of $1 \mathrm{UI} /$ hour $(0.475 \mu \mathrm{g} / \mathrm{h})$, reproducing "normal" conditions $(1,31)$. The effectiveness of the PTH supplementation was subsequently assessed by blood ionized Ca measurement. In addition, all TPTX rats were given $2 \mu \mathrm{g} .100 \mathrm{~g} \mathrm{BW}^{-1}$ of L-thyroxine (Fluka) in drinking water daily (32). The same experiment was then repeated, but this time in TPTX rats without any PTH supplementation. Finally, a subset of TPTX, PTH-supplemented rats were treated by subcutaneous injections of pamidronate (2.5 $\mu \mathrm{g} . \mathrm{g} \mathrm{BW}^{-1}$, daily for 4 days prior to the experiment and throughout the remainder of the experiment) and were given either NPS2143 or the vehicle alone during 9 days.

At steady state, urine collection was performed daily under mineral oil in the urine collector for electrolyte measurements. Urine creatinine (modified kinetic Jaffé's colorimetric method) and phosphate were measured with a Konelab i20 auto-analyzer (Thermo Scientific). Urinary Ca concentration was measured by atomic absorption spectrophotometry (PerkinElmer, model AA-3110). Plasma creatinine concentration was measured by ion exchange chromatography; urinary $\mathrm{Na}^{+}$and $\mathrm{K}^{+}$concentrations were measured by flame photometry (IL943). Ionized Ca was measured with an ABL $77 \mathrm{pH} /$ blood gas analyzer (Radiometer). The level of deoxypyridinoline crosslink was measured using a chemiluminescence assay on an Immulite 2000 automated analyzer (DPC Siemens Medical Solutions Diagnostics). Values are reported relative to creatinine concentrations. Plasma osteocalcin was measured using ECLIA (Roche Diagnostics). Rat plasma PTH concentration was determined using ELISA (Immutopics). Stool was also collected from baseline to the end of the study. Stool samples collected daily were desiccated at $50^{\circ} \mathrm{C}$ overnight and stored at $-80^{\circ} \mathrm{C}$ until analysis. Each 24-hour fecal sample was dissolved in $25 \mathrm{ml}$ nitric acid $(1.5 \mathrm{M})$ at $95^{\circ} \mathrm{C}$ for 12 hours. The solution was brought to a total volume of $25 \mathrm{ml}$ with deionized water, and Ca was measured.

\section{$m R N A$ extraction and $R T$ real-time $P C R$ analysis}

RNA was extracted according to the technique previously described (33) from nephron segments. RNA was reverse transcribed in $20 \mu \mathrm{l}$ using the first-strand cDNA synthesis kit for RT-PCR (Roche Diagnostics), according to the manufacturer's protocol. Real-time PCR was performed on a LightCycler (Roche Diagnostics) with the LightCycler FastStart DNA Master SYBR Green I kit (Roche Diagnostics) according to the manufacturer's protocols, except that the reaction volume was reduced 2.5 -fold. Specific primers were designed using ProbeDesign 2 (Roche Diagnostics). No DNA was detectable in samples that did not undergo reverse transcription or in blanks run without cDNA. In each experiment, a standardization curve was made using serial dilutions of standard cDNA stock solutions made from rat whole kidney RNA, and the amount of PCR product was calculated as a percentage of the standard DNA. Results are expressed as mean \pm SEM from 5-6 rats. The presence of the mRNA encoding CaSR along the nephron was determined by RT-PCR. The $5^{\prime}$ (sense 5'-TAGGAGGTCTTCCTATCCATTT-3', antisense 5'-GGCTGCTGTTTATCTCCTCTA-3') and $3^{\prime}$ (sense 5'-CAGCCAGCTTTGGCTTAC-3', antisense $5^{\prime}$-CGGGAGATGTTGGGACG-3') primers were designed from rat Casr (GenBank 24247).

\section{Cell culture}

Cell culture HEK293 cells were maintained in DMEM supplemented with $10 \%$ fetal bovine serum and $1 \%$ penicillin/streptomycin. For DNA transfection, cells were grown to $60 \%-70 \%$ confluence on plastic culture dishes and then transiently transfected for 5 hours with plasmids using the Lipofectamine plus kit according to manufacturer's instructions (Invitrogen).

\section{Antibodies}

Abs against Tamm-Horsfall protein (Santa Cruz Biotechnology Inc.) (34), aquaporin-2 (Santa Cruz Biotechnology Inc.) (35), and $\gamma$-ENaC (36) have all been characterized and reported previously. The anti-rabbit polyclonal $\mathrm{Ab}$ to the thiazide-sensitive $\mathrm{NaCl}$ cotransporter $(\mathrm{Ncc})$, a gift from D.H. Ellison (Oregon Health and Science University, Portland, Oregon, USA), has been previously characterized $(37,38)$, as well as the $\mathrm{Ab}$ to pendrin (gift from P. Aronson, Yale University School of Medicine, New Haven, Connecticut, USA) $(39,40)$. Anti- $\mathrm{Na}^{+}, \mathrm{K}^{+}$-ATPase $\alpha_{1}$ subunit $\mathrm{Ab}$ was a gift from F. Jaisser (Cordeliers Research Centre, Paris, France) (41).

Anti-CaSR Abs used were MA1-934, a mouse mAb product from Affinity BioReagents corresponding to residues 214-235 of human CaSR (42); and the rabbit polyclonal anti-CaSR (Ab10-12) directed against the C-terminal tail of the rat CaSR generated against a 116-amino-acid polypeptide starting from amino acid 961 of the rat CaSR and fused to GST. The fusion protein was purified, and $100 \mu \mathrm{g}$ was injected into a rabbit to generate the Ab10-12 antiserum as previously described (43).

The specificity of both Abs was assessed by immunoprecipitation experiments after transfection of rat CaSR (Supplemental Figure 1). Absorption tests for immunoperoxidase staining were also performed in rat kidney (see Supplemental Figure 2 for more details). As shown in Supplemental Figure $2 \mathrm{~A}$, the tissue section was incubated with the rabbit polyclonal anti-CaSR $\mathrm{Ab}$. As shown in Supplemental Figure 2B, the Ab was preincubated with the immunizing peptide $(100 \mu \mathrm{g} / \mathrm{ml})$.

\section{Western blotting and immunolabeling}

Crude membrane fractions were isolated from whole rat renal cortex as described previously (44). All immunohistochemistry experiments were performed exactly as previously detailed $(38,45-47)$. The affinity-purified anti-CaSR Abs were diluted (1:500 and 1:700 for Ab10-12 and MA1-934, respectively) in background reducing buffer and applied for 1 hour at room temperature. Kidney sections were also double-labeled with antiTamm-Horsfall protein, anti-Ncc, and anti-aquaporin-2 using streptavidin-cyanine-2 (Amersham Pharmacia Biotech, diluted 1:1,000) and Alexa Fluor 568 (Invitrogen, diluted 1:800).

For EM experiments, ultrathin cryosections $-80 \mathrm{~nm}$ thick, incubated with Ab10-12 and subsequently with goat anti-rabbit 10-nm gold particles (BB International) - were examined in a Philips CM 100 electron microscope.

\section{Protein preparation, immunoblotting, and immunoprecipitation}

Forty-eight hours after transfection, cells were washed 3 times with cold PBS and lysed in 0.2- or 0.5-ml lysis buffer (120 mM Tris-HEPES, pH 7.4; $150 \mathrm{mM} \mathrm{NaCl}, 5 \mathrm{mM}$ EDTA, $3 \mathrm{mM} \mathrm{KCl} ; 1 \%$ [v/v] Triton X-100) containing protease inhibitors (Complete, Roche 1697498). Samples were harvested, sonicated, and centrifuged at $140,000 \mathrm{~g}$ for 15 minutes at $4^{\circ} \mathrm{C}$. Protein expression levels were assessed after normalizing and loading equal amounts of total protein for 7.5\% SDS-PAGE separation and Western blotting with anti-CaSR Abs raised against the $\mathrm{C}$-terminal or the $\mathrm{N}$-terminal region of the receptor. For immunoprecipitation, cells were solubilized with lysis buffer containing $0.4 \mathrm{M} \mathrm{NaCl}$; $0.5 \mathrm{mM}$ EGTA; $1.5 \mathrm{mM} \mathrm{MgCl}$; 10 mM HEPES, pH 7.9; 5\% (v/v) glycerol; 0.5\% (v/v) Nonidet P-40; and protease inhibitors (Complete, Roche). Immunoprecipitation was carried out using anti-CaSR Abs, followed by affinity purification using protein G-agarose beads (Dynal). After incubation with protein G-agarose beads for 1 hour at room temperature, the immunocomplex was washed 3 times in PBS (Invitrogen). The protein samples were boiled in loading buffer, run on gradient $6 \%-20 \%$ SDS-polyacrylamide gels, probed with primary Abs of interest and horseradish peroxidase-conjugated secondary $\mathrm{Ab}$, according to standard procedures. Proteins were visualized by enhanced chemiluminescence detection (PerkinElmer) following the manufacturer's instructions. 


\section{In vitro microperfusion}

TALs and CCDs were isolated and microperfused in vitro as previously described (48-50). The functional expression of CaSR was assessed in cTAL and CCD by studying the effect of two distinct CaSR activators on cytosolic Ca concentration $\left(\left[\mathrm{Ca}^{2+}\right]_{i}\right)$ : (a) a polyvalent cation (neomycin) that binds to the extracellular $\mathrm{N}$-terminal $\mathrm{CaSR}$ domain, mimics $\mathrm{Ca}^{2+}$, then activates CaSR (51); and (b) NPS-R568, a hydrophobic low-molecular-weight drug that allosterically activates $\operatorname{CaSR}(52,53)$. The technique used for $\left[\mathrm{Ca}^{2+}\right]_{\mathrm{i}}$ measurement in single microdissected tubules has been described (54). Finally, the effect of NPS2143 on transepithelial $\mathrm{Ca}^{2+}, \mathrm{Na}^{+}$, and $\mathrm{Cl}^{-}$transport in the cTAL was studied. Experiments were successively performed on each tubule for 3 periods: initial, experimental ( $1 \mu \mathrm{M}$ NPS2143 addition in the peritubular fluid), and recovery. Four samples of tubular fluid were collected during each period.

\section{Measurement of transepithelial Ca absorption $\left(J_{C a}\right)$}

The tubules were equilibrated for $20-30$ minutes at $37^{\circ} \mathrm{C}$ in the initial perfusion and bath solutions, and the luminal flow was adjusted to 2.5-4 nl/min. The collection time $(t)$ for each sample was recorded, and the sample volume $(v)$ was measured using volumetric pipettes, in order to calculate the perfusion rate $(V=v / t)$. Tubule length $(L)$ was measured using the optical scale of the microscope. Ca concentration was measured in 10- to 12-nl samples of perfused and collected fluid using Fluo-3 and the flow-through microfluorometer NanoFlo apparatus (World Precision Instruments) (55). The Fluo-3 was reconstituted in HPLC-grade water and diluted $1 / 6(\mathrm{v} / \mathrm{v})$ in phosphate buffer, $\mathrm{pH} 7.3$.

Ca chloride calibrating solutions $(0.5,1$, and $2 \mathrm{mM})$ were prepared using $10 \mathrm{mM}$ MOPS buffer, $100 \mathrm{mM} \mathrm{KCl}$, and $1 \mathrm{mM} \mathrm{MgCl}_{2}$ solution adjusted to $\mathrm{pH} 7.3$ with $\mathrm{NaOH}$. $\mathrm{J}_{\mathrm{Ca}}$ was calculated using the equation: $J_{\mathrm{Ca}}=\left([\mathrm{Ca}]_{\mathrm{i}}-\right.$ $\left.[\mathrm{Ca}]_{\circ}\right) \times V / L$, where $[\mathrm{Ca}]_{i}$ is the concentration of $\mathrm{Ca}$ in the perfusate, $[\mathrm{Ca}]_{\mathrm{o}}$ is the concentration of $\mathrm{Ca}$ in the collected fluid, $V$ is the collection rate (in $\mathrm{nl} / \mathrm{min}$ ), and $L$ is the tubule length (in $\mathrm{mm}$ ). An averaged net transepithelial Ca flux was calculated for each period in a given tubule.

Measurement of transepithelial sodium chloride absorption $\left(J_{N a}, J_{C l}\right)$ Tubule perfusion and sample collection were performed as described above. Measurements of Na concentration were performed by HPLC. The Dionex DX-500 system (Dionex Corp.) consisted of an AS50 autosampler, a GP50 gradient pump, and an ED40 electrochemical detector $\left(\mathrm{Na}^{+}\right)$. The signal-to-noise ratio of the conductivity measurement was enhanced by employing a cation self-regenerating suppressor (CSRS-ultra, $4 \mathrm{~mm}$ ) that was set in the auto-suppression recycle mode. The HPLC column consisted of a Dionex IonPac CS12 column $(4 \times 250 \mathrm{~mm})$ equipped with a guard column CG12A $(4 \times 50 \mathrm{~mm})$. The mobile phase consisted of $18 \mathrm{mM}$ methanesulfonic acid. Tubular fluid, perfusion solution, and standard solutions were sampled under mineral oil with a calibrated pipette (about $12 \mathrm{nl}$ ) and transferred in a vial containing $35 \mu \mathrm{l}$ of mobile phase of the HPLC with $\mathrm{LiNO}_{3}$ as internal standard. Peaks of $\mathrm{Na}^{+}$were adjusted with the value of the $\mathrm{Li}^{+}$internal standard, to limit the variations due to automatic injection. In each experimental run, perfusion and bath solutions were tested in 4 or 5 replicates, and the reproducibility of the measure was evaluated: coefficient of variation $(\mathrm{CV})<0.03$ for $\mathrm{Na}^{+}$determination. In each experimental run, a calibration curve was tested: correlation coefficients $>0.98$.

Chloride was measured by microcoulometry as previously described (49). Four to 6 aliquots (about $0.5 \mathrm{nl}$ ) of collected fluid taken during each period, perfusion solution, and standard solutions were put on the silver plate of the titrator. Each sample was titrated until $240 \mathrm{mV}$ of potential with a pipette filled with $\mathrm{AgNO}_{3}$-agar. The value of the capacity used for each titration was compared with the calibration curve to obtain the chloride content of the sample. The mean of each collection was used. Reproducibility of measurements was high: $\mathrm{CV}<0.03$. In each experimental run, a calibration curve was tested: correlation coefficients $>0.98$.

\section{Calculation of the rate of absorption of $\mathrm{Na}^{+}$and $\mathrm{Cl}^{-}$}

For each collection, ion flux $(J)$ was calculated and corrected for the length of the tubule: $J_{\mathrm{Na}}=\left([\mathrm{Na}]_{\mathrm{i}}-[\mathrm{Na}]_{\mathrm{o}}\right) * V / L ; J_{\mathrm{Cl}}=\left([\mathrm{Cl}]_{\mathrm{i}}-[\mathrm{Cl}]_{\mathrm{o}}\right) * V / L$. Therefore, positive values indicate net absorption of the ion. For each tubule, the mean of the 4 collections was used per period.

\section{Measurement of Vte}

Vte was measured with a DP-301 differential electrometer (Warner Instrument Corp.) by use of a $\mathrm{Ag} / \mathrm{AgCl}$ electrode connected to the perfusion pipette via a $0.15-\mathrm{M} \mathrm{NaCl}$ agar bridge; a $0.15-\mathrm{M} \mathrm{NaCl}$ agar bridge also connected the peritubular bath to an $\mathrm{Ag} / \mathrm{AgCl}$ electrode. Vte was measured during each period at the tip of the perfusion pipette.

\section{Measurement of transepithelial permeability to $\mathrm{Ca}$}

The basic approach used to determine Ca permeability involved construction of a transepithelial gradient of $\mathrm{NaCl}$ (56). To clamp for Vte, asymmetric solutions were used: the tubule was bathed with a solution containing $144 \mathrm{mmol} / \mathrm{l} \mathrm{Na}$ and $120 \mathrm{mmol} / \mathrm{l} \mathrm{Cl}$, whereas the lumen was perfused with a solution containing $53 \mathrm{mmol} / 1 \mathrm{Na}$ and $29 \mathrm{mmol} / \mathrm{l} \mathrm{Cl}$. The resulting bath-to-lumen $\mathrm{NaCl}$ concentration gradient thus created generated a constant and stable transepithelial potential (Vte). The recorded Vte was corrected for liquid junction potential. In addition, furosemide $(100 \mu \mathrm{M})$ was applied to the luminal side of the TAL to inhibit active transports. Assuming that $\mathrm{Ca}$ transport was passive, that there was no significant net water flux, that the transtubular Ca concentration was negligibly small, and that the net flux was linearly related to the voltage, the paracellular pathway permeability to $\mathrm{Ca}(P)$ can be calculated using the relation $J=P z C F E / R T$, where $J$ is the net transepithelial transport, $z$ is the valence, $C$ is the Ca concentration, $F$ is Faraday's number, $E$ is the Vte, $R$ is the gas constant, and $T$ is the absolute temperature.

As detailed above, during these experiments, the cTAL permeability to Ca was determined during 3 periods of experiments successively performed on each tubule: initial, experimental ( $1 \mu \mathrm{M}$ NPS2 143 in the peritubular fluid), and recovery.

\section{Cytosolic Ca measurement}

As shown in Supplemental Figure 6, cTAL and CCD cells were loaded with the fluorescent probe Fura-2 (Molecular Probes, Invitrogen) and prepared as a $10-\mathrm{mM}$ Fura-2 AM stock in DMSO by exposing the cells for approximately 20 minutes at room temperature to the control bath solution. Double wavelength measurements of Fura-2 fluorescence were carried out after a 30-minute equilibration period with a standard photometric set up (model MSP 21) assisted by a microcomputer (51). As a rule, all tubules were studied within less than 1 hour following their microdissection. Bath solution was delivered at a rate of $20 \mathrm{ml} / \mathrm{min}$ and warmed to $37 \pm 0.5^{\circ} \mathrm{C}$ by water jacket immediately upstream to the chamber. The perfusion rate was adjusted by hydrostatic pressure to approximately $20 \mathrm{nl} / \mathrm{min}$ to prevent axial changes in the composition of luminal fluid.

\section{Solution compositions}

The standard medium used for cytosolic Ca measurement experiments was (in $\mathrm{mM}$ ): $\mathrm{NaCl} 141 ;$ Ca lactate $1 ; \mathrm{K}_{2} \mathrm{HPO}_{4} 2 ; \mathrm{Na}$ citrate $1 ; \mathrm{MgSO}_{4} 1.2 ; \mathrm{CaCl}_{2} 1$; D-glucose 5.5; HEPES 10; pH 7.4. Osmolality was $300 \mathrm{mOsm} / \mathrm{kg}$. The solution for flux measurements $\left(\mathrm{J}_{\mathrm{Ca}}, \mathrm{J}_{\mathrm{Na}}, \mathrm{J}_{\mathrm{Cl}}\right.$ ) was (in $\mathrm{mM}$ ): $\mathrm{NaCl} 116$; Ca lactate 2; $\mathrm{K}_{2} \mathrm{HPO}_{4} 2 ; \mathrm{Na}$ citrate $1 ; \mathrm{MgSO}_{4} 1.2 ; \mathrm{CaCl}_{2} 1$; D-glucose 5.5; HEPES 10; alanine 5; $\mathrm{NaHCO}_{3} 23 ; \mathrm{pH} 7.4$. The solutions used for paracellular perme- 
ability measurements were as follows (in $\mathrm{mM}$ ): bath solution, $\mathrm{NaCl} 118$, Ca lactate 1 , glucose $5.5, \mathrm{NaHCO}_{3} 23$, alanine $5, \mathrm{~K}_{2} \mathrm{HPO}_{4} 2, \mathrm{CaCl}_{2} 1$, HEPES 10 , $\mathrm{MgSO}_{4} 1.2$, Na citrate 1; perfusion (lumen) solution, $\mathrm{NaCl} 27$, Ca lactate 1, mannitol 182.2, glucose 5.5, $\mathrm{NaHCO}_{3} 23$, alanine 5, $\mathrm{K}_{2} \mathrm{HPO}_{4} 2, \mathrm{CaCl}_{2} 1$, HEPES 10, $\mathrm{MgSO}_{4} 1.2, \mathrm{Na}$ citrate 1.

The solutions containing bicarbonate were continuously gassed with $95 \% \mathrm{O}_{2} / 5 \% \mathrm{CO}_{2}$ at $37^{\circ} \mathrm{C}$. Before each experiment, osmolality and $\mathrm{pH}$ were measured in bulk solutions. Osmolality was $300 \mathrm{mOsm} / \mathrm{kg}$. Alanine and HEPES were obtained from Research Organics; all other chemicals were from Sigma-Aldrich.

\section{Statistics}

Values are expressed as mean \pm SEM. Continuous variables were analyzed by Mann-Whitney $U$ test or non-parametric Wilcoxon matched-pairs signed-rank test, as appropriate. All tests were 2 -sided. All statistical analyses were performed using Stata 11.0 software (StataCorp.). Probability values of 0.05 or less were regarded as statistically significant.

\section{Study approval}

All experimental procedures were performed in accordance with the Guide for the Care and Use of Laboratory Animals (NIH publication no. 93-23, revised 1985 ) and with the French Government animal welfare policy. All experi- ments were approved (CEEA34.PH.02912) by the Ethical Committee for Animal Tests, Ile de France-Paris Descartes (Université Paris-Descartes).

\section{Acknowledgments}

Part of this study was funded by an Agence Nationale de la Recherche grant (ANR-07-PHYSIO-027). A. Loupy was funded by a grant from the Société de Néphrologie and Amgen. S.K. Ramakrishnan was funded by Erasmus Mundus External Cooperation Window Lot 15 India. B. Wootla has been funded by an ANR grant (ANR-07PHYSIO-027). We thank Sylvie Demaretz and Jose Rodriguez for their technical expertise, Theano Irinopoulou for excellent work in confocal analysis, and Gilles Morineau for help in measuring bone remodeling markers. Abs to thiazide-sensitive $\mathrm{NaCl}$ cotransporter and pendrin were gifts from D. Ellison and P. Aronson, respectively.

Received for publication February 24, 2011, and accepted in revised form June 28, 2012.

Address correspondence to: Pascal Houillier, Service de Physiologie, Hôpital Européen Georges Pompidou, 20, rue Leblanc, F-75015 Paris, France. Phone: 33.1.5609.3972; Fax: 33.1.5609.2675; E-mail: pascal.houillier@egp.aphp.fr.
1. Jaeger $P$, et al. Animal model of primary hyperparathyroidism. Am J Physiol. 1987;252(6 pt 1):E790-E798.

2. Brown EM, et al. Cloning and characterization of an extracellular $\mathrm{Ca}(2+)$-sensing receptor from bovine parathyroid. Nature. 1993;366(6455):575-580.

3. Riccardi D, Brown EM. Physiology and pathophysiology of the calcium-sensing receptor in the kidney. Am J Physiol Renal Physiol. 2010; 298(3):F485-F499.

4. Brown EM. Clinical lessons from the calcium-sensing receptor. Nat Clin Pract Endocrinol Metab. 2007; 3(2):122-133.

5. Peacock M, Bilezikian JP, Klassen PS, Guo MD, Turner SA, Shoback D. Cinacalcet hydrochloride maintains long-term normocalcemia in patients with primary hyperparathyroidism. JClin Endocrinol Metab. 2005;90(1):135-141.

6. Block GA, et al. Cinacalcet for secondary hyperparathyroidism in patients receiving hemodialysis. NEngl J Med. 2004;350(15):1516-1525.

7. Tu Q, Pi M, Karsenty G, Simpson L, Liu S, Quarles LD. Rescue of the skeletal phenotype in CasRdeficient mice by transfer onto the Gcm 2 null background. J Clin Invest. 2003;111(7):1029-1037.

8. Kos $\mathrm{CH}$, et al. The calcium-sensing receptor is required for normal calcium homeostasis independent of parathyroid hormone. J Clin Invest. 2003; 111(7):1021-1028

9. Egbuna O, et al. The full-length calcium-sensing receptor dampens the calcemic response to 1alpha,25(OH)2 vitamin D3 in vivo independently of parathyroid hormone. Am J Physiol Renal Physiol. 2009;297(3):F720-F728.

10. Kantham L, et al. The calcium-sensing receptor (CaSR) defends against hypercalcemia independently of its regulation of parathyroid hormone secretion. Am J Physiol Endocrinol Metab. 2009; 297(4):E915-E923.

11. Gowen M, et al. Antagonizing the parathyroid calcium receptor stimulates parathyroid hormone secretion and bone formation in osteopenic rats. J Clin Invest. 2000;105(11):1595-1604.

12. Jara A, Lee E, Stauber D, Moatamed F, Felsenfeld AJ, Kleeman CR. Phosphate depletion in the rat: effect of bisphosphonates and the calcemic response to PTH. Kidney Int. 1999;55(4):1434-1443.

13. Lee CT, Huynh VM, Lai LW, Lien YH. Cyclosporine A-induced hypercalciuria in calbindin-D28k knockout and wild-type mice. Kidney Int. 2002; 62(6):2055-2061.

14. Brown EM, MacLeod RJ. Extracellular calcium sensing and extracellular calcium signaling. Physiol Rev. 2001;81(1):239-297.

15. Yang T, Hassan S, Huang YG, Smart AM, Briggs JP, Schnermann JB. Expression of PTHrP, PTH/ $\mathrm{PTHrP}$ receptor, and $\mathrm{Ca}(2+)$-sensing receptor mRNAs along the rat nephron. Am J Physiol. 1997; 272(6 pt 2):F751-F758

16. Braden GL, Sharon GC, Marconi TC. Immunolocalization of the calcium-sensing receptor in developing and adult human kidney [ASN abstract PO673]. J Am Soc Nephrol. 2009;20:269A. http:// www.asn-online.org/education_and_meetings/ kidneyweek/archives.

17. Ho C, et al. A mouse model of human familial hypocalciuric hypercalcemia and neonatal severe hyperparathyroidism. Nat Genet. 1995;11(4):389-394.

18. Parfitt AM. Equilibrium and disequilibrium hypercalcemia: new light on an old concept. Metab Bone Dis Rel Res. 1979;1:279-293.

19. Maruani G, Hertig A, Paillard M, Houillier P. Normocalcemic primary hyperparathyroidism: evidence for a generalized target-tissue resistance to parathyroid hormone. JClin Endocrinol Metab. 2003; 88(10):4641-4648.

20. Law WM, James EM Jr, Charboneau JW, Purnell DC, Heath H. High-resolution parathyroid ultrasonography in familial benign hypercalcemia (familial hypocalciuric hypercalcemia). Mayo Clin Proc. 1984;59(3):153-155.

21. Abugassa S, Nordenstrom J, Jarhult J. Bone mineral density in patients with familial hypocalciuric hypercalcaemia (FHH). EurJ Surg. 1992;158(8):397-402.

22. Sands JM, et al. Apical extracellular calcium/ polyvalent cation-sensing receptor regulates vasopressin-elicited water permeability in rat kidney inner medullary collecting duct. J Clin Invest. 1997; 99(6):1399-1405

23. Levi M, Peterson L, Berl T. Mechanism of concentrating defect in hypercalcemia. Kidney Int. 1983; 23(3):489-497.

24. Earm JH, et al. Decreased aquaporin-2 expression and apical plasma membrane delivery in kidney collecting ducts of polyuric hypercalcemic rats. J Am Soc Nephrol. 1998;9(12):2181-2193.

25. Renkema KY, et al. The calcium-sensing receptor promotes urinary acidification to prevent nephrolithiasis. J Am Soc Nephrol. 2009;20(8):1705-1713.

26. De Jesus Ferreira MC, Bailly C. Extracellular Ca2+ decreases chloride reabsorption in rat CTAL by inhibiting cAMP pathway. Am J Physiol. 1998; 275(2 pt 2):F198-F203.

27. Desfleurs E, et al. Calcium-sensing receptor: regulation of electrolyte transport in the thick ascending limb of Henle's loop. Kidney Blood Press Res. 1998; 21(6):401-412.

28. Wittner M, Mandon B, Roinel N, de Rouffignac C, Di Stefano A. Hormonal stimulation of $\mathrm{Ca} 2+$ and $\mathrm{Mg} 2+$ transport in the cortical thick ascending limb of Henle's loop of the mouse: evidence for a change in the paracellular pathway permeability. Pflugers Arch. 1993;423(5-6):387-396

29. Motoyama HI, Friedman PA. Calcium-sensing receptor regulation of PTH-dependent calcium absorption by mouse cortical ascending limbs. Am J Physiol Renal Physiol. 2002;283(3):F399-F406.

30. Tan SQ, Thomas D, Jao W, Bourdeau JE, Lau K. Surgical thyroparathyroidectomy of the rabbit. Am J Physiol. 1987;252(4 pt 2):F761-F767.

31. Ibrahim MM, Forte LR, Thomas ML. Maintenance of normocalcemia by continuous infusion of the synthetic bovine parathyroid hormone (1-34) in parathyroidectomized rats. Calcif Tissue Int. 1982; 34(6):553-557.

32. Mohebbi N, Kovacikova J, Nowik M, Wagner CA. Thyroid hormone deficiency alters expression of acid-base transporters in rat kidney. Am J Physiol Renal Physiol. 2007;293(1):F416-F427.

33. Cheval L, et al. Atlas of gene expression in the mouse kidney: new features of glomerular parietal cells. Physiol Genomics. 2011;43(3):161-173.

34. Bourgeois S, et al. Differentiated thick ascending limb (TAL) cultured cells derived from SV40 transgenic mice express functional apical NHE2 isoform: effect of nitric oxide. Pflugers Arch. 2003; 446(6):672-683.

35. Duong Van Huyen JP, et al. GDF15 triggers homeostatic proliferation of acid-secreting collecting duct cells. J Am Soc Nephrol. 2008;19(10):1965-1974.

36. Masilamani S, Kim GH, Mitchell C, Wade JB, Knepper MA. Aldosterone-mediated regulation of ENaC alpha, beta, and gamma subunit proteins in rat kidney. J Clin Invest. 1999;104(7):R19-R23.

37. Chambrey $R$, et al. Immunolocalization of the 
$\mathrm{Na}+/ \mathrm{H}+$ exchanger isoform NHE2 in rat kidney. Am J Physiol. 1998;275(3 pt 2):F379-F386.

38. McCulloch F, Chambrey R, Eladari D, Peti-Peterdi J. Localization of connexin 30 in the luminal membrane of cells in the distal nephron. Am J Physiol Renal Physiol. 2005;289(6):F1304-F1312.

39. Quentin F, et al. The Cl-/HCO3- exchanger pendrin in the rat kidney is regulated in response to chronic alterations in chloride balance. Am J Physiol Renal Physiol. 2004;287(6):F1179-F1188.

40. Knauf F, Yang CL, Thomson RB, Mentone SA, Giebisch G, Aronson PS. Identification of a chloride-formate exchanger expressed on the brush border membrane of renal proximal tubule cells. Proc Natl Acad Sci U S A. 2001;98(16):9425-9430.

41. Jaisser F. [Molecular and functional diversity of NA,K-ATPase and renal H,K-ATPases]. Nephrologie. 1996;17(7):401-408.

42. Goldsmith PK, et al. Expression, purification, and biochemical characterization of the amino-terminal extracellular domain of the human calcium receptor. J Biol Chem. 1999;274(16):11303-11309.

43. Petrel C, et al. Modeling and mutagenesis of the binding site of Calhex 231, a novel negative alloste- ric modulator of the extracellular $\mathrm{Ca}(2+)$-sensing receptor. J Biol Chem. 2003;278(49):49487-49494.

44. Quentin F, et al. Regulation of the Cl-/HCO3exchanger AE2 in rat thick ascending limb of Henle's loop in response to changes in acid-base and sodium balance. J Am Soc Nephrol. 2004; 15(12):2988-2997.

45. Chambrey R, et al. Localization and functional characterization of $\mathrm{Na}+/ \mathrm{H}+$ exchanger isoform NHE4 in rat thick ascending limbs. Am J Physio Renal Physiol. 2001;281(4):F707-F717.

46. Eladari D, et al. Expression of RhCG, a new putative $\mathrm{NH}(3) / \mathrm{NH}(4)(+)$ transporter, along the rat nephron. J Am Soc Nephrol. 2002;13(8):1999-2008.

47. Quentin F, et al. RhBG and RhCG, the putative ammonia transporters, are expressed in the same cells in the distal nephron. J Am Soc Nephrol. 2003; 14(3):545-554

48. Burg M, Grantham J, Abramow M, Orloff J. Preparation and study of fragments of single rabbit nephrons. Am J Physiol. 1966;210(6):1293-1298.

49. Lerolle N, Bourgeois S, Leviel F, Lebrun G, Paillard $\mathrm{M}$, Houillier P. Angiotensin II inhibits $\mathrm{NaCl}$ absorption in the rat medullary thick ascending limb. AmJ
Physiol Renal Physiol. 2004;287(3):F404-F410.

50. Picard N, et al. Defective ENaC processing and function in tissue kallikrein-deficient mice. J Biol Chem. 2008;283(8):4602-4611.

51. Champigneulle A, Siga E, Vassent G, ImbertTeboul M. V2-like vasopressin receptor mobilizes intracellular $\mathrm{Ca} 2+$ in rat medullary collecting tubules. Am J Physiol. 1993;265(1 pt 2):F35-F45.

52 . Nemeth EF, et al. Calcimimetics with potent and selective activity on the parathyroid calcium receptor. Proc Natl Acad Sci U S A. 1998;95(7):4040-4045.

53. Harrington PE, Fotsch C. Calcium sensing receptor activators: calcimimetics. Curr Med Chem. 2007; 14(28):3027-3034.

54. Poumarat JS, et al. The luminal membrane of rat thick limb expresses AT1 receptor and aminopeptidase activities. Kidney Int. 2002;62(2):434-445.

55. Zhelyaskov VR, Liu S, Broderick MP. Analysis of nanoliter samples of electrolytes using a flowthrough microfluorometer. Kidney Int. 2000; 57(4):1764-1769.

56. Bourdeau JE, Burg MB. Voltage dependence of calcium transport in the thick ascending limb of Henle's loop. Am J Physiol. 1979;236(4):F357-F364. 